\title{
Nuevas perspectivas acerca del colapso Moche en el Bajo Jequetepeque
}

Resultados preliminares de la segunda campaña de investigación del proyecto arqueológico Cerro Chepén

Nouvelles perspectives concernant l'effondrement de la culture Moche dans le Bas Jequetepeque. Résultats préliminaires de la deuxième campagne de recherche du projet archéologique Cerro Chepén

New perspectives about the late Moche collapse in the lower Jequetepeque Valley. Preliminary results of the second field season of the Cerro Chepen archaeological project

\section{Marco Rosas Rintel}

\section{(2) OpenEdition}

\section{Journals}

Edición electrónica

URL: http://journals.openedition.org/bifea/3835

DOI: $10.4000 /$ bifea.3835

ISSN: 2076-5827

Editor

Institut Français d'Études Andines

Edición impresa

Fecha de publicación: 1 agosto 2007

Paginación: 221-240

ISSN: 0303-7495

Referencia electrónica

Marco Rosas Rintel, « Nuevas perspectivas acerca del colapso Moche en el Bajo Jequetepeque », Bulletin de l'Institut français d'études andines [En línea], 36 (2) | 2007, Publicado el 01 febrero 2008, consultado el 10 diciembre 2020. URL : http://journals.openedition.org/bifea/3835 ; DOI : https:// doi.org/10.4000/bifea.3835

\section{(c)}

Les contenus du Bulletin de l'Institut français d'études andines sont mis à disposition selon les termes de la licence Creative Commons Attribution - Pas d'Utilisation Commerciale - Pas de Modification 4.0 International. 


\title{
Nuevas perspectivas acerca del colapso Moche en el Bajo Jequetepeque Resultados preliminares de la segunda campaña de investigación del proyecto arqueológico Cerro Chepén
}

\author{
Marco Rosas Rintel*
}

\begin{abstract}
Resumen
Evidencia arqueológica recuperada en el asentamiento fortificado Moche Tardío más extenso del Valle Bajo del Jequetepeque - Cerro Chepen — sugiere que una situación de «presión externa» fue responsable del colapso político de las comunidades Moche Tardío asentadas en la región. Excavaciones conducidas en una serie de edificios de élite ubicados en el Sector Monumental de Cerro Chepén descubrieron una curiosa asociación de cerámica doméstica de estilo Moche Tardío y cerámica fina de élite de estilo Cajamarca Cursivo Floral en los espacios internos de estas estructuras. Considerando esta evidencia en conjunción con el estilo arquitectónico de los edificios excavados, el esmerado diseño defensivo del Sector Monumental de Cerro Chepén, el patrón de asentamiento de las comunidades Moche Tardío del Valle del Jequetepeque, y una serie de fechados radiocarbónicos que ubican el final de la ocupación de Cerro Chepén en las postrimerías de la Fase Moche Tardío, se esboza un modelo de colapso político para las comunidades locales del valle que tiene como principal agente una irrupción militar concebida y ejecutada por líderes de la etnia Cajamarca.
\end{abstract}

Palabras clave - Cerro Chepén, fase Moche Tardío, arquitectura grupo-patio, cultura Cajamarca, objetos de prestigio, colapso político

\footnotetext{
* Universidad de Nuevo México, Departamento de Antropología, Programa Doctoral de Arqueología. Este artículo fue escrito mientras el autor se desempeñaba como profesor de arqueología en la Pontificia Universidad Católica del Perú. E-mail: mrosas@unm.edu.
} 


\title{
Nouvelles perspectives concernant l'effondrement de la culture Moche dans le Bas Jequetepeque Résultats préliminaires de la deuxième campagne de recherche du projet archéologique Cerro Chepén
}

\section{Résumé}

L'évidence archéologique récupérée à l'emplacement fortifié de l'époqueMoche Tardío le plus étendu de la vallée basse du Jequetepeque —Cerro Chepén — suggère que l'exercice d'une «pression externe» fut à l'origine de l'effondrement politique des communautés Moche Tardío établies dans la région. Des fouilles réalisées dans une série de bâtiments d'élite situés dans le Secteur Monumental de Cerro Chepén ont permis de découvrir une association curieuse de céramique domestique de style Moche Tardío et de céramique fine d'élite de style Cajamarca Cursivo Floral dans les espaces internes de ces structures. Si I'on considère cette évidence en conjonction avec: le style architectural de ces bâtiments fouillés, le plan défensif travaillé avec soin du Secteur Monumental de Cerro Chepén, le modèle de l'emplacement des communautés Moche Tardío de la vallée du Jequetepeque et une série d'objets datés au carbone radioactif qui situent la fin de l'occupation de Cerro Chepén dans la dernière période de la Fase Moche Tardío, s'ébauche un modèle d'effondrement politique pour les communautés locales de la vallée provoqué essentiellement par une irruption militaire conçue et exécutée par les leaders de l'ethnie Cajamarca.

Mots clés - Cerro Chepén, phase Moche Tardío, architecture groupe-patio, culture Cajamarca, objets de prestige, effondrement politique

\section{New perspectives about the late Moche collapse in the lower Jequetepeque Valley. Preliminary results of the second field season of the Cerro Chepen archaeological project}

\begin{abstract}
Archaeological evidence recovered at the largest Late Moche fortified settlement in the Lower Jequetepeque Valley —Cerro Chepén— suggests that a situation of «external pressure» was responsible for the political collapse of the Late Moche communities of the region. Excavations conducted at a series of elite residences located in the Monumental Sector of Cerro Chepen revealed a curious association between Late Moche domestic wares and fine Cajamarca-style elite ceramics within the interior spaces of these structures. An evaluation of this evidence in conjunction with the architectural style of the buildings, the neat defensive design of Cerro Chepen's Monumental Sector, the known settlement pattern of the Late Moche communities in the Jequetepeque Valley, and a series of C-14 dates that place the end of the occupation of Cerro Chepen roughly at the close of the Late Moche Phase, leads to the tentative conclusion that a military incursion undertaken by Cajamarca leaders was the main causal factor for the disappearance of the local Late Moche political systems.
\end{abstract}

Key words - Cerro Chepen, Late Moche Phase, patio-group architecture, Cajamarca culture, prestige objects, political collapse 


\section{INTRODUCCIÓN}

Este artículo tiene como propósito presentar los avances de investigación del Proyecto Arqueológico Cerro Chepén (PROCECHE), después de completada la temporada de excavaciones del año 2004. El PROCECHE es un proyecto multidisciplinario que busca entender los mecanismos que llevaron al colapso a los sistemas políticos Moche asentados en el valle bajo del Jequetepeque. Para cumplir este objetivo, el PROCECHE ha emprendido la investigación de un extenso sitio habitacional fortificado - Cerro Chepén - ubicado centralmente dentro del cono aluvial de este valle norteño. El sitio es significativo no solo por su posición central, sino también por la magnitud de sus fortificaciones o por ser el asentamiento del periodo Moche Tardío más extenso dentro de la sección norte del valle. Su característica más saltante es la curiosa configuración de algunas de sus estructuras monumentales que integran ambientes de estilo serrano en su diseño estructural. Este tipo de ambientes — galerías, algunas de dos pisos, rodeando patios cuadrangulares - es extraño a la tradición arquitectónica de la costa norte peruana, siendo, en cambio, comúnmente asociados a la tradición arquitectónica Huari, que experimentó una amplia expansión por la sierra peruana entre los siglos VIII y IX de nuestra era. En el año 2003, el PROCECHE emprendió un programa de excavaciones de estas estructuras con el fin de recuperar evidencia material que nos permitiera determinar de manera precisa la identidad cultural de sus ocupantes. Confiábamos que esta evidencia brindaría una respuesta no solo al desmesurado arreglo defensivo del sitio, sino también a las condiciones que generaron el colapso de las entidades políticas Moche de la región.

El presente artículo expone los resultados de las excavaciones conducidas durante el año 2004. Durante esta temporada, que resultó ser particularmente exitosa, se excavó un gran edificio erigido, siguiendo modelos arquitectónicos altoandinos, que es interpretado tentativamente como una estructura residencial de élite (Edificio IV). Las excavaciones han evidenciado una inesperada asociación entre cerámica doméstica Moche Tardío y cerámica fina de la sierra de Cajamarca al interior de los patios, galerías y cuartos que componen la estructura. La evidencia recuperada en este edificio sugiere la presencia de funcionarios serranos en el corazón del Sector Monumental de Cerro Chepén. Esta presencia habría ostentado una posición de dominio, pues el Edificio IV se yergue en una de las ubicaciones más preferenciales del sitio.

La presencia de un posible enclave fortificado altoandino en el corazón de un territorio Moche ofrece una perspectiva interesante acerca de las condiciones que generaron el colapso de los sistemas políticos Moche Tardío que ocuparon el bajo Jequetepeque. En concordancia con el modelo de colapso político propuesto por Joseph Tainter (1988), que es adoptado como sustento teórico de esta investigación, sostengo que la irrupción foránea habría incrementado los costos de manutención de las diversas entidades políticas Moche Tardío locales, a la vez que restaba importantes recursos que habrían sido necesarios para su eventual recuperación. En el caso específico del bajo Jequetepeque, esta intrusión habría tenido un efecto colateral inesperado: el debilitamiento interno del mismo ente agresor. El enclave foráneo centrado en Cerro Chepén habría desaparecido al mismo tiempo y por las mismas razones que generaron el deterioro de los sistemas políticos surgidos en el valle. Se postula que el escenario cultural del bajo Jequetepeque durante el periodo Moche Tardío ilustra vívidamente los eventos que se suscitan cuando dos poderes regionales se enfrentan en una guerra de desgaste de largo aliento.

Este artículo está organizado en cuatro secciones. En la primera presento el marco teórico general que guía esta investigación, y los modelos que han sido propuestos para explicar el colapso político Moche Tardío, resaltando sus elementos comunes y su conexión con la teoría antropológica de colapso cultural. A continuación, introduzco el caso específico de Cerro Chepén. En esta sección presento una detallada descripción del sitio, en la que resalto su posición dominante dentro del valle, y el esmerado diseño defensivo de su Sector Monumental. La tercera sección está dedicada al Edificio IV, una estructura residencial de élite enclavada en el corazón del Sector Monumental de Cerro Chepén. Aquí presento los resultados de las excavaciones desarrolladas 
durante el año 2004 y expongo la evidencia arqueológica que me lleva a plantear la existencia de una ocupación altoandina en el corazón monumental de Cerro Chepén. Finalmente, cierro el artículo con una propuesta de colapso para las sociedades Moche Tardío del Bajo Jequetepeque, que está inspirada en los inesperados hallazgos del 2004.

Los estudios desarrollados en Cerro Chepén ofrecen un escenario de colapso para las poblaciones asentadas en el bajo Jequetepeque que es totalmente singular dentro del ámbito Moche Tardío. Este nuevo escenario debe ser tomado en cuenta a la hora de proponer modelos organizativos para esta insigne tradición cultural que ocupó distintos valles de la Costa Norte durante los siglos VI, VII y VIII de nuestra era. Indudablemente, modelos que postulan la existencia de un único estado centralizado deben ser abandonados. La diversidad de la experiencia cultural Moche Tardío debe ser, por el contrario, abordada valle por valle e inclusive considerando sectores de algunos valles por separado. Dentro de cada uno de estos espacios, las formaciones políticas Moche Tardío habrían desarrollado distintas propuestas organizativas, y padecido distintas condiciones conducentes al colapso cultural.

\section{COLAPSO CULTURAL Y COLAPSO MOCHE}

La teoría antropológica moderna interpreta el colapso de formaciones políticas pasadas como el producto de la disolución de los lazos económicos e ideológicos que unen a la casta gobernante con la población base (Yofee, 1979; Tainter, 1988; Bawden, 1995; Cowgill, 1995: 259; Kaufman, 1995: 223). Esta perspectiva ha sido planteada de manera elocuente por el arqueólogo norteamericano Joseph Tainter (1988) en un modelo titulado «los retornos marginales decrecientes de la complejidad política». Este modelo sugiere que, conforme una sociedad avanza hacia un estado de alta complejidad sociopolítica, se alcanza un punto en el que los beneficios que obtiene la población base por mantener las instituciones centrales de poder se ven largamente excedidos por los costos de esta inversión (1988: 93). Tainter sostiene que una vez que los costos de mantenimiento de los órganos centrales del estado comienzan a reportar un retorno marginal decreciente, la base popular puede considerar el status quo improductivo y simplemente optar por dejar de aportar subsidios económicos (tributos) al estado.

Tainter (1988) y otros (Yofee, 1979; Trigger, 1990; Cowgill, 1995; Berdan \& Smith, 1996) han detectado una serie de factores que tienden a incrementar los costos de manutención de sistemas políticos complejos, disminuyendo concurrentemente la calidad de vida de la gente común. Entre estos factores se cuentan los altos costos de la agricultura intensiva, la proliferación burocrática, el derroche asociado a actividades de legitimación de poder, sistemas ineficientes de redistribución de recursos, y la alta demanda energética de la especialización económica y política. Si bien la mayoría de estos factores son de naturaleza económica, también pueden existir profundas incongruencias ideológicas al interior de una sociedad que pueden incrementar las distancias entre gobernados y gobernantes. Una de esas incongruencias podría haber afectado a la sociedad Moche (Bawden, 1994; 1995; 1996; 2001).

Bawden habla de una paradoja estructural al interior de la sociedad Moche, en la que la naturaleza exclusiva del poder de las élites entraba en contradicción con principios andinos tradicionales que promovían la integración comunal a través de la reciprocidad, el parentesco y el culto a los ancestros (Bawden, 1995: 258; 1996: 223-224). Esta paradoja representó una fuerza disruptiva latente al interior de la sociedad Moche, y puede haber sido exacerbada una vez que la gente común percibió que las instituciones de gobierno retribuían muy poco en comparación con lo que demandaban para funcionar.

Precisamente, tres modelos de colapso que han sido propuestos para la cultura Moche inciden en esta falla ideológica. En Pampa Grande, la ciudad más grande de la sección Norte del territorio Moche, Shimada (1994) halló evidencia de un final violento asociado a una revuelta popular. Las élites Moche locales, tozudamente fieles a principios tradicionales, habrían sido incapaces de convocar ideológicamente a un segmento no-Moche de la población que creció en número 
y capacidad organizativa a lo largo del tiempo. En Galindo, la ciudad más septentrional del territorio Moche Tardío, el final también estuvo acompañado de una rebelión, esta vez gestada en respuesta a las medidas coercitivas extremas que implementaron los gobernantes locales (Bawden, 1982a; 1996). Estas medidas represivas, que entre otras cosas se reflejan en la erección de un gran muro que aisló los barrios populares del resto de la ciudad, fueron implementadas para contrarrestar el fracaso de una revisión ideológica que rechazó símbolos tradicionales de poder. Parcialmente relacionado al argumento de Bawden se halla la propuesta de Luis Jaime Castillo $(1993 ; 2000 ; 2003)$ para explicar el ocaso de las élites Moche Tardío del sitio de San José de Moro. Según Castillo, las élites del sitio cayeron en descrédito al emprender un programa de revitalización ideológica, que implicó la asimilación de los símbolos de poder de una formación cultural —la cultura Huari- que gozaba de mucho prestigio en la época. El nuevo programa ideológico no habría ofrecido a las clases populares una justificación convincente sobre las grandes distancias sociales que las separaba de la nobleza privilegiada.

Dos propuestas adicionales de colapso, no relacionadas con factores ideológicos, han sido planteadas para el caso Moche. Estas propuestas tienen en común el hecho de resaltar la ingerencia de factores no económicos - perturbaciones ambientales y guerra- en procesos de colapso cultural. Estos factores también tienen la propiedad de afectar negativamente la calidad de vida de la gente común, menoscabando su capacidad de aportar económicamente a las instituciones centrales de poder. Cuando estos factores se combinan con los productos negativos colaterales de procesos de complejización sociopolítica, las fuerzas disruptivas que se generan al interior de sociedades pueden ser prácticamente incontenibles.

El argumento de perturbaciones ambientales como factor detonante del colapso Moche surge de estudios realizados entre mediados de 1970 y 1980 en glaciares de la cordillera andina (Thompson, 1980; Thompson \& Mosley-Thompson, 1987; ver también Thompson et al., $1979 ; 1984 ; 1985 ; 1986)$. Muestras de hielo obtenidas del barrenado profundo de glaciares permitieron armar un registro detallado de precipitaciones de 1500 años de duración. Este registro evidencia un patrón de inestabilidad ambiental durante prácticamente toda la extensión del periodo Moche Tardío (ca. 550-800 d. C.), caracterizado por la sucesión de varias sequías severas, interrumpidas por un único episodio de humedad ambiental excesiva (Shimada et al., 1991: 261; Shimada, 1994: 249). Evidentemente, este periodo de inestabilidad climática habría afectado la capacidad productiva de las comunidades Moche asentadas en la costa. Es probable que se alcanzara un punto en el que la base productiva de la sociedad simplemente fuera incapaz de aportar los recursos que demandaba la clase dirigente, generándose un cisma insalvable. Un argumento similar, basado en deterioro ambiental, ha sido propuesto para explicar el colapso de las sociedades del periodo Clásico Maya asentadas en las tierras bajas del Petén (Santley, Killion \& Lycett, 1986; Culbert, 1995).

Finalmente, la teoría de conflicto externo surge de un análisis de patrones de asentamiento desarrollado por Tom Dillehay (2001) en el valle bajo del Jequetepeque, región que acoge a los sitios de Cerro Chepén y San José de Moro. Dillehay observó que, durante el periodo Moche Tardío, el mapa político del bajo Jequetepeque estaba caracterizado por una situación de fraccionamiento total. Durante este periodo, la población local vivía dentro de o en la cercanía inmediata de unos pocos asentamientos fortificados o con fortificaciones, la mayoría de tamaño intermedio (20 a 40 ha), estratégicamente dispuestos ascendiendo la falda de cerros. El panorama arqueológico del valle alude, pues, a una situación de «conflicto esporádico o posiblemente guerra organizada entre comunidades» (Dillehay, 2001: 271). Dillehay asume que el conflicto surgió como resultado de la competencia que las comunidades sostenían por los mejores terrenos de cultivo (2001: 275).

En el caso del conflicto armado, el colapso no surge únicamente del total aniquilamiento de comunidades locales por parte de un vecino poderoso. Puede que las comunidades sean capaces de resistir de manera eficaz la arremetida de fuerzas beligerantes, pero esto tampoco quiere decir que podrán sobrevivir indefinidamente. La necesidad de enfrentar una campaña militar siempre impondrá serias presiones sobre las economías internas, que se irán agravando 
conforme se alargue el conflicto. Si la población local no está preparada para suplir tanto las cuotas de tributo ordinarias, como los cupos especiales de guerra impuestos por los líderes locales, el escenario de colapso interno es inminente.

En conclusión, son varias las perspectivas que han sido usadas para abordar el tema del colapso Moche. Todas aluden a escenarios locales, sugiriendo que el territorio Moche Tardío estuvo dividido en distintas entidades políticas regionales, aparentemente centradas en valles individuales. En todas las propuestas, el factor predisponente de colapso es el alto costo de mantenimiento de sistemas políticos complejos. Este costo, sin embargo, no tiene porque ser medida suficiente de ruina política. Si los líderes locales son capaces de implementar una maquinaria ideológica capaz de contrarrestar las tendencias independentistas de la población, el status quo podrá ser mantenido exitosamente. Es cuando el programa ideológico falla, o cuando nuevos factores (deterioro ambiental, guerra) se suman para agravar las presiones económicas impuestas sobre la base social, que todo el sistema político derrumbará.

Pasemos ahora a revisar el caso específico de Cerro Chepén, y a examinar la evidencia que sugiere un nuevo escenario de conflicto político al interior del valle del Jequetepeque.

\section{CERRO CHEPÉN}

El sitio arqueológico de Cerro Chepén es, en muchos aspectos, un asentamiento típico del periodo Moche Tardío en el valle del Jequetepeque. Se ciñe al patrón de asentamientos Moche Tardío descubierto por Dillehay (2001), en el sentido de representar un gran sitio habitacional

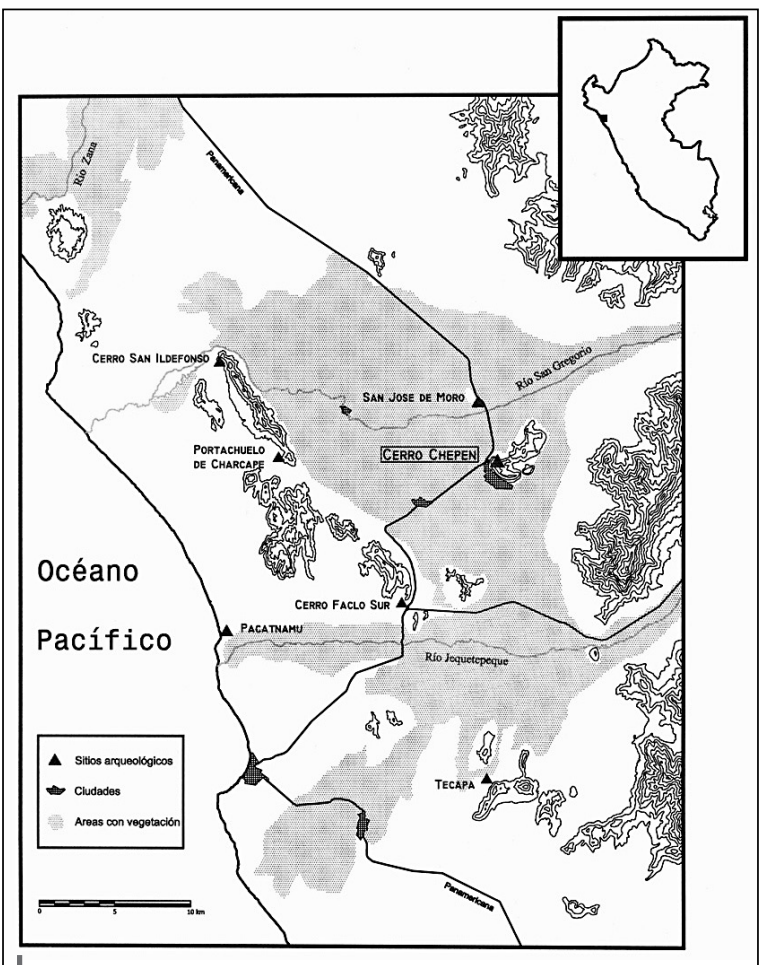

Figura 1 - Mapa del Valle Bajo del Jequetepeque mostrando la ubicación de Cerro Chepén y de otros importantes sitios Moche Tardío nucleado (cerca de 40 ha de extensión), fortificado, y estratégicamente ubicado sobre la cima y faldas orientales de un gran cerro. Sin embargo, Cerro Chepén se distingue de otros asentamientos contemporáneos en su ubicación preferencial, la magnitud de sus obras defensivas, y la monumentalidad de sus construcciones internas.

Por un lado, el sitio se yergue prácticamente en el centro del gran cono aluvial que forman los ríos Jequetepeque y San Gregorio en su aproximación al mar (fig. 1). Desde esta ubicación preferencial, los antiguos habitantes del sitio ejercían control visual de los puntos neurálgicos del valle bajo, que incluyen:

- prácticamente toda la expansión de terreno agrícola al norte del curso del río Jequetepeque;

- los «cuellos» de los valles del Río Chamán y del Río Jequetepeque;

- las laderas áridas de los cerros que circundan la mayor concentración de terreno fértil en el llano aluvial.

Esta ubicación también les confirió a los habitantes del sitio la ventaja adicional de tener acceso directo a terrenos agrícolas que actualmente presentan alto rendimiento productivo, y que antiguamente habrían sido 
irrigados con aguas de los canales Serrano y Chepén (Eling, 1987: 256-257, 445). El sitio mismo se ubica a solo 3,5 km al sur del cauce actual del río San Gregorio que, si bien se «seca» durante

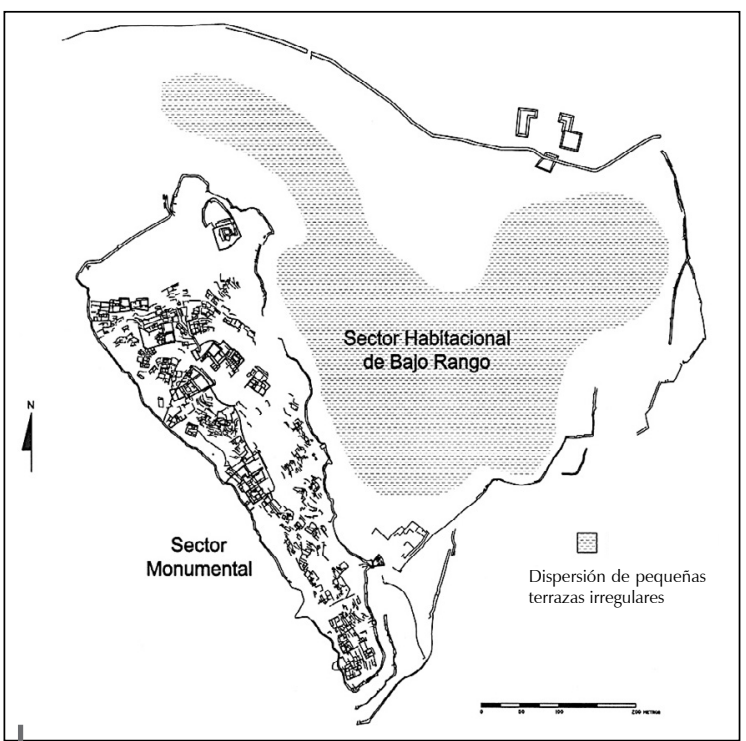

Figura 2 - Plano general de Cerro Chepén los meses invernales, presenta numerosos bolsones permanentes de agua subterránea asociados.

La magnitud de las obras defensivas del sitio es otro rasgo que merece ser resaltado. El sitio arqueológico de Cerro Chepén consta de dos sectores claramente definidos por sendas murallas perimétricas (fig. 2). El primer sector, que denominamos monumental, se ubica a lo largo de la parte más alta del cerro. Este sector ocupa aproximadamente la cuarta parte de la extensión total del sitio (9,7 ha) y tiene forma de cuña alargada. Es especialmente interesante la muralla que protege a este sector, la cual ha sido construida en su totalidad con piedras extraídas del mismo cerro. La muralla tiene un largo continuo de $1717 \mathrm{~m}$, un grosor promedio de cabecera de $2,5 \mathrm{~m}$, y llega a alcanzar una altura de 5,5 m en algunos sectores. El diseño de la muralla sigue un rígido plan defensivo, con solo tres accesos sumamente resguardados, un total de once torreones distribuidos, sobre todo, a lo largo de su trazo este, y hasta once acumulaciones de pequeños cantos rodados (interpretados como municiones de huaracas). Esta muralla es, sin lugar a dudas, la obra defensiva más colosal de la región, siendo solamente comparable con la muralla externa de la ciudad de Pacatnamú, la cual, sin embargo, data del periodo Chimú (Donnan, 1986: 54).

La segunda muralla bordea el segundo sector de ocupación del sitio, que se extiende sobre las laderas orientales del cerro, al este de la gran muralla defensiva. Este sector está compuesto casi exclusivamente por pequeñas terrazas interpretadas tentativamente como viviendas de la gente común. La muralla perimétrica que protege al sector habitacional de bajo rango es de menor altura y grosor que la antes mencionada. Llama también la atención la ausencia de torreones y concentraciones de cantos rodados en asociación con el segundo muro. Un dato interesante acerca de este muro es que, en las secciones construidas sobre o cerca al llano, tanto adobes como piedra fueron utilizados en su construcción.

Finalmente, Cerro Chepén se distingue también por la monumentalidad de sus edificaciones. Todas las construcciones monumentales del sitio se concentran al interior del denominado Sector Monumental. Se distinguen claramente nueve grandes edificios y algunos conjuntos arquitectónicos menores, de carácter periférico (fig. 3). De entre los primeros, cuatro edificios (IV, VI, VIII y IX) se destacan por ostentar una posición preferencial dentro del Sector Monumental. En primer lugar, están construidos sobre la cima del cerro, lo que significa que sus ocupantes ejercían un control visual sobre todo el sitio. En segundo lugar, fueron construidos en el centro del Sector monumental. Esto quiere decir que estas estructuras son las más distantes de cualquiera de los accesos ubicados en la muralla perimétrica. En otras palabras, dentro de un sector diseñado para defensa, estas estructuras ocupan la mejor posición defensiva. Es innegable que sus ocupantes gozaron de privilegios no accesibles al común de los habitantes de Cerro Chepén.

Un estudio más cercano de estos cuatro edificios nos permitió identificar un tercer elemento que los singulariza frente a los demás edificios del sitio, y probablemente de todo el valle bajo del Jequetepeque (Swenson, 2004: 404). Las cuatro estructuras mencionadas son las únicas que adoptan en su diseño arquitectónico galerías, a veces rodeando, a veces flanqueando, patios 


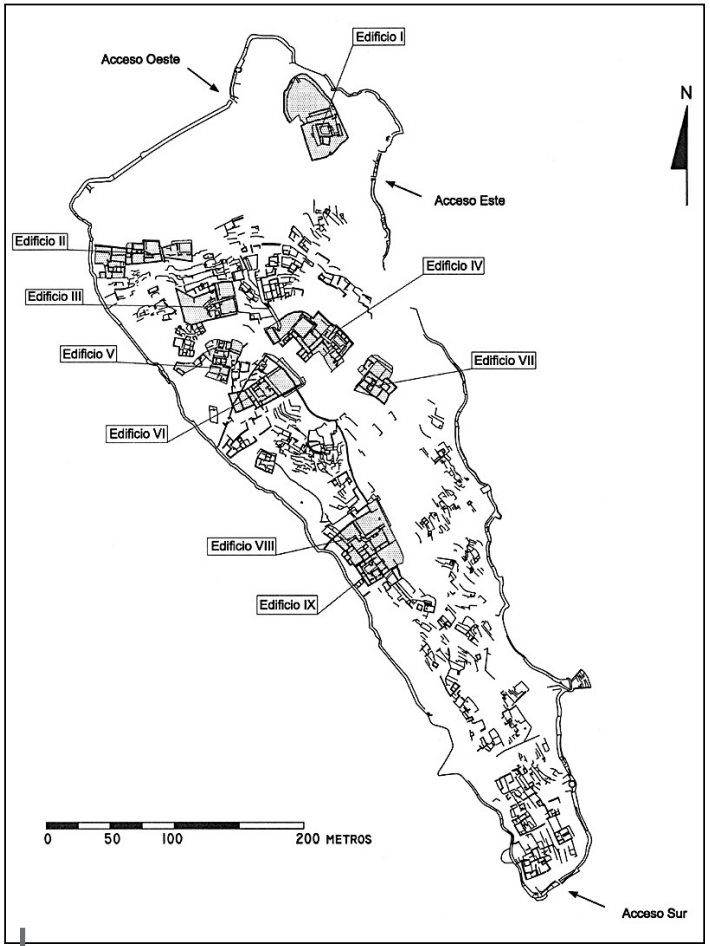

Figura 3 - Plano del Sector Monumental de Cerro Chepén mostrando ubicación de edificios y accesos en la muralla perimétrica

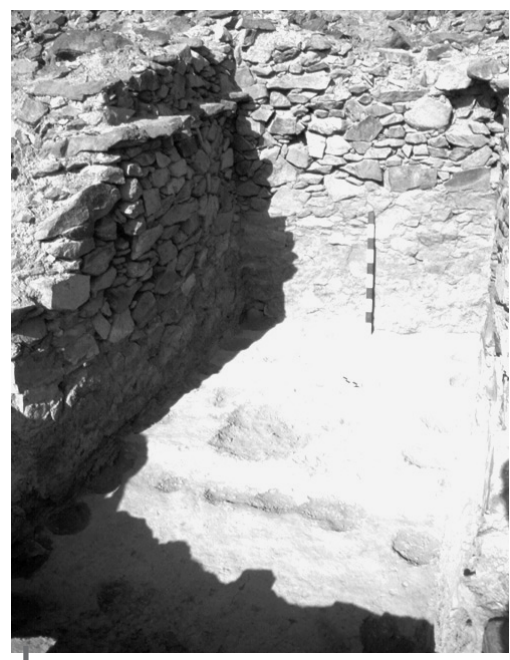

Figura 4 - Excavación de una galería de dos pisos en el Edificio VIII

Nótese la fila de ménsulas en la pared cuadrangulares. Algunas galerías tienen ménsulas que indican la existencia de un segundo piso (figura 4). Este tipo de arquitectura es absolutamente ajeno a la tradición constructiva costeña de la época siendo típico, por el contrario, de tradiciones altoandinas. Algunos autores llaman a este modelo arquitectónico «grupo-patio» y lo consideran característico de la tradición constructiva ayacuchana de Huari, originada aproximadamente en el siglo VII de nuestra era (Brewster-Wray, 1983; Anders, 1986; Isbell, 1989; 1991; Schreiber, 1992). Otros autores, sin embargo, lo relacionan con tradiciones arquitectónicas más tempranas de la sierra Norte (Topic \& Lange Topic, 1982-1985).

Los cuatro edificios centrales del Sector Monumental de Cerro Chepén ofrecen evidencia arquitectónica que sugiere la presencia de pobladores altoandinos en el valle bajo del Jequetepeque. A mi entender, esta presencia habría tenido una injerencia directa en los eventos que llevaron al colapso a los sistemas políticos locales del valle. Se hacía necesario, pues, buscar evidencia adicional que pudiera corroborar la existencia de huestes serranas en el cerro. En el año 2003 se inició un programa de excavación de edificios monumentales en el sitio en busca de esta evidencia. Una de las estructuras excavadas fue el Edificio IV. Como se verá en las páginas siguientes, el Edificio IV reportó numerosos ejemplos de objetos de prestigio - expresados en cerámica fina - relacionados con la etnia altoandina de Cajamarca. Se puede ahora sugerir un modelo de colapso basado en presión externa. Este modelo será abordado en la parte final de este artículo.

\section{LA EXCAVACIÓN DEL EDIFICIO IV}

El Edificio IV, excavado durante la campaña del 2004, es una de las estructuras más llamativas de Cerro Chepén. Con $1645 \mathrm{~m}^{2}$ de área, es el segundo edificio más extenso del Sector Monumental del sitio. El Edificio IV se distingue, también, por su elaborado diseño arquitectónico. Una elegante gradería, de 23 metros de largo y 2 metros de ancho, representa el acceso formal al edificio. Esta gradería corta la terraza frontal de la estructura, que está delineada por un muro en el que se alternan piedras grandes y pequeñas en aparejo ordenado. De hecho, los 
muros de contención que definen las terrazas del edificio son tan altos — hasta 4,9 metrosque son fácilmente visibles desde distintos sectores del sitio e inclusive desde el nivel del valle cultivado.

Al interior del edificio se ubican espacios de distinta forma y arreglo arquitectónico. La estrategia de excavación desplegada en el Edificio IV nos llevó a sondear los espacios más significativos de la estructura. Estos espacios incluyen:

- un gran patio cuadrangular cercado por un grueso muro exornado con hornacinas;

- tres cuartos interconectados (interpretados como habitaciones);

- una galería de dos pisos con patio anexo;

- un conjunto de cuartos y galerías dispuestos alrededor de un patio respetando el típico arreglo de «grupo-patio»;

- una zona de arquitectura irregular, en la parte posterior del edificio (fig. 5).

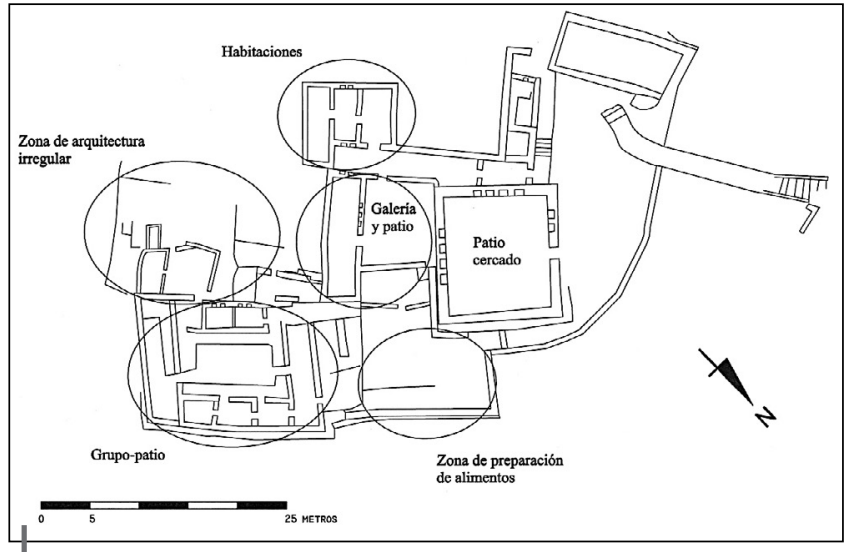

Figura 5 - Organización funcional de ambientes en el Edificio IV

Las excavaciones persiguieron tres fines: entender la secuencia constructiva del edificio, definir la función de espacios, e indagar acerca de la identidad cultural de los ocupantes de la estructura.

En lo que toca al primer punto, descubrimos que la gran obra, que nosotros llamamos Edificio IV, fue originalmente erigida sobre terreno virgen. En otras palabras, el edificio no fue construido sobre un espacio que ocupaba una estructura más antigua. El edificio tampoco es una construcción que fue creciendo paulatinamente en el tiempo. Su planta actual respondió a un plan maestro que fue concebido y ejecutado de una sola vez. En cuanto al momento de construcción de la obra, todas las unidades de excavación reportaron ingentes cantidades de cerámica doméstica de estilo Moche Tardío (fig. 6). Estos fragmentos procedían tanto de los rellenos constructivos más antiguos (incluyendo el relleno de los muros mismos), como de los últimos pisos de ocupación. Se puede, entonces, concluir con seguridad que la obra fue construida y ocupada durante el periodo Moche Tardío.

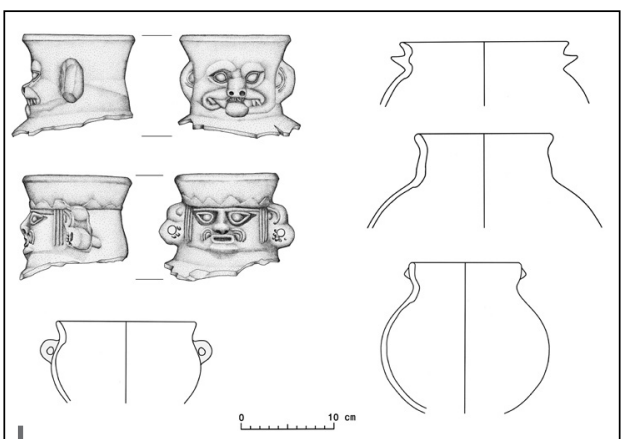

Figura 6 - Ejemplos de cerámica doméstica Moche Tardío hallada en los edificios del Sector Monumental de Cerro Chepén
Los estudios de función de ambientes corrieron con distinta suerte en distintos sectores. Las excavaciones ofrecieron identificaciones positivas en el caso del gran patio cercado, los tres cuartos interconectados y la zona de arquitectura irregular. El primero es, a todas luces, un espacio de reuniones oficiales. Su diseño repite los elementos propiosa los patios ceremoniales representados en maquetas de barro del periodo Moche Tardío (Castillo et al., 1997), y maquetas similares de madera pertenecientes a la posterior época Chimú (Donnan, 1975; Uceda, 1997). Los tres cuartos interconectados han sido interpretados como habitaciones en función de su ubicación dentro del edificio, arreglo arquitectónico y rasgos asociados. La zona de arquitectura irregular, por otro lado, habría funcionado como una zona industrial 
donde artesanos, posiblemente actuando bajo la estricta supervisión de las élites, habrían elaborado adornos e implementos personales de distintos materiales. De los ambientes excavados en este sector proceden los pocos ejemplos de materias primas (prills de cobre, conchas marinas), desechos de producción (conchas cortadas, desechos de talla lítica) y utensilios y herramientas de trabajo (una gran «mesa» de piedra, percutores, espátulas de hueso, pulidores de cerámica) reportados en el edificio. Finalmente, afloramientos superficiales de carbones y tierra quemada nos permitieron ubicar una posible zona de preparación de alimentos sobre una terraza que se abre al este de la estructura (fig. 5). En conclusión, el carácter monumental de la estructura, aunada a la diversidad de funciones que cumplieron sus espacios interiores (entre los que se cuentan ambientes destinados a habitación), nos permite concluir con seguridad que el Edificio IV habría funcionado como una residencia de élite.

La galería de dos pisos y el «grupo-patio», dos conjuntos arquitectónicos construidos respetando cánones altoandinos, no ofrecieron información útil que pudiera resolver el dilema funcional. Sí reportaron, por el contrario, información significativa que ayudó a solucionar el tercer objetivo trazado por la investigación: lograr la identificación cultural de los ocupantes.

La categoría de cultura material que escogimos en nuestra investigación para determinar la identidad cultural de los ocupantes fue la de objetos de prestigio. El término «objetos de prestigio» alude a los bienes manufacturados, ligeros y transportables, que los líderes de sociedades complejas emplean para señalar su alta posición social. Estos bienes generalmente poseen una carga ideológica muy fuerte que identifica a sus poseedores como a individuos con derecho a ejercer poder sobre otros (DeMarrais et al., 1996: 26). Los líderes locales siempre mostraron una marcada preocupación por evitar que estos objetos, que encarnan auténticos símbolos de poder, caigan en manos de personas no autorizadas. Este objetivo lo consiguieron de dos maneras distintas: a) monopolizando la producción y distribución de estos bienes, y b) usando materiales exóticos, de difícil acceso, y/o tecnología y mano de obra altamente especializada en su confección (Brumfiel \& Earle, 1990). Estas precauciones terminan confiriendo a los objetos características únicas, que los convierten en piezas muy llamativas, irreplicables, generalmente decoradas con la iconografía distintiva de los círculos de poder de la tradición cultural correspondiente.

Entre las antiguas culturas peruanas, la cerámica fina fue uno de los medios comúnmente más usados para la reproducción de objetos de prestigio. En el caso particular de la cultura Moche, por ejemplo, evidencia iconográfica indica que los líderes locales generalmente hacían ostentación de finas piezas cerámicas cuando presidían ceremonias públicas (fig. 7). Evidencia arqueológica testifica, por otro lado, que las élites usaron y descartaron estas piezas en sus

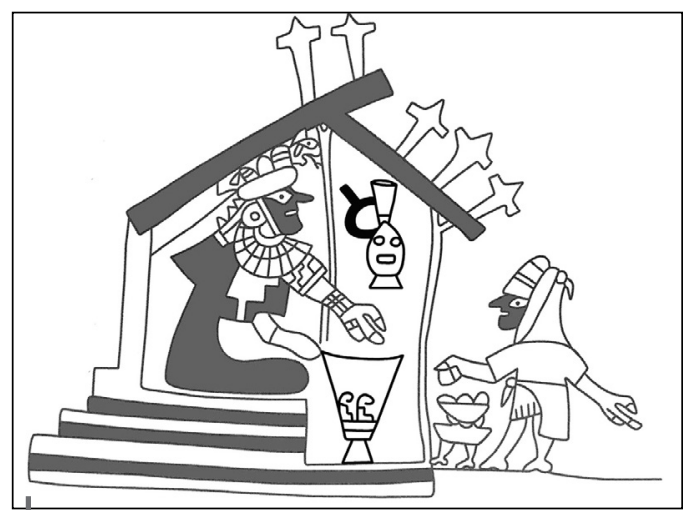

Figura 7 - Detalle de la «Escena del banquete»: dignatario Moche exhibe, entre otras insignias de poder, dos finas piezas cerámicas

Redibujado de Larco Hoyle, 2001 [1939]: figura 212 edificios de habitación (Bawden, 1982b: 175; Shimada, 1994: 169). Las mismas piezas han sido halladas formando parte de los ricos ajuares funerarios de individuos notables (Strong, 1947; Donnan \& Castillo, 1992; 1994; Alva \& Donnan, 1993; Narváez, 1994; Donnan, 2003; Tello et al., 2003). En todos los casos, y no solamente aquellos relacionados con la cultura Moche, las finas piezas cerámicas usadas como objetos de prestigio presentan un alto grado de elaboración en su confección, siendo su estilo y decoración altamente distintivos de tradición cultural. Estas piezas se convertían, pues, en un medio ideal para tentar la identificación de los ocupantes del Edificio IV.

La galería de dos pisos, el «grupo-patio» y una de los espacios identificados como habitaciones reportaron ingentes cantidades 
de fragmentos de cerámica fina que en muchas ocasiones pudieron ser unidos permitiéndonos restituir las vasijas casi a su estado original. Estos fragmentos procedían de hoyos con basura cavados en los pisos, o de basurales superficiales que periódicamente fueron sellados bajo distintos pisos de ocupación. Lo más curioso acerca de estas vasijas es la virtual ausencia del estilo más representativo de la tradición Moche Tardío: las finas botellas de asa-estribo decoradas con línea fina (Donnan \& McClelland, 1999). La muestra está dominada (98\%), por el contrario, por estilos que son asignables a etnias alto-andinas.

La muestra de vasijas finas recuperadas del Edificio IV y de los dos edificios excavados durante la temporada del 2003 ha sido organizada en cuatro alfares en función del color, forma y decoración de las piezas. Estos alfares han sido denominados «Cajamarca Cursivo Floral», «Cajamarca decorado con línea roja», «Alfar anaranjado pulido» y «Alfar negro pulido». Un análisis de Número Mínimo de Vasijas, establecido a partir de fragmentos de bordes y vasijas parcialmente reconstruidas, nos ha permitido determinar la popularidad relativa de cada uno de estos alfares dentro de la muestra total de vasijas finas $(\mathrm{N}=205)$.

El alfar más distintivo de la muestra está encarnado por finos platos de caolín propios al estilo Cursivo Floral de la Tradición Cajamarca Medio (fig. 8). Estos platos componen el 7,8 \% de nuestra muestra. Su estilo representativo está bien definido en la literatura especializada (Reichlen \& Reichlen, 1970; Terada \& Matsumoto, 1985; Julien, 1988). Sabemos con precisión que este estilo se desarrolló en el valle de Cajamarca aproximadamente entre los años 450 y 900 d. C. (Matsumoto, 1994: 185).

El alfar «Cajamarca decorado con línea roja», representando otro $7,8 \%$ de la muestra, abarca exclusivamente a platos con base anular, que denotan indistintamente pasta de caolín o pasta roja (fig. 9). La decoración se circunscribe al interior del plato e implica diseños lineares trazados con un pigmento rojo. Platos de estas características también han sido hallados en San José de Moro tanto en contextos Moche Tardío (Castillo \& Donnan, 1994: figura 3. 31), como en contextos del periodo Transicional (Rucabado \& Castillo, 2003: figura 1. 3). Si bien algunos de estos platos han sido denominados «Cajamarca Costeño» en el pasado (Rucabado \& Castillo, 2003: 24), la presencia de especimenes de caolín en nuestra muestra asociaría propiamente a este alfar con tradiciones serranas.

El alfar «Anaranjado pulido» aparenta ser exclusivo al sitio de Cerro Chepén dentro del valle bajo del Jequetepeque. Este alfar compone el $22,9 \%$ de la muestra y abarca exclusivamente a escudillas de base plana o ligeramente curva (fig. 10). Si bien la gran mayoría de especimenes carece de decoración, unos pocos muestran diseños lineares trazados con pintura negra orgánica

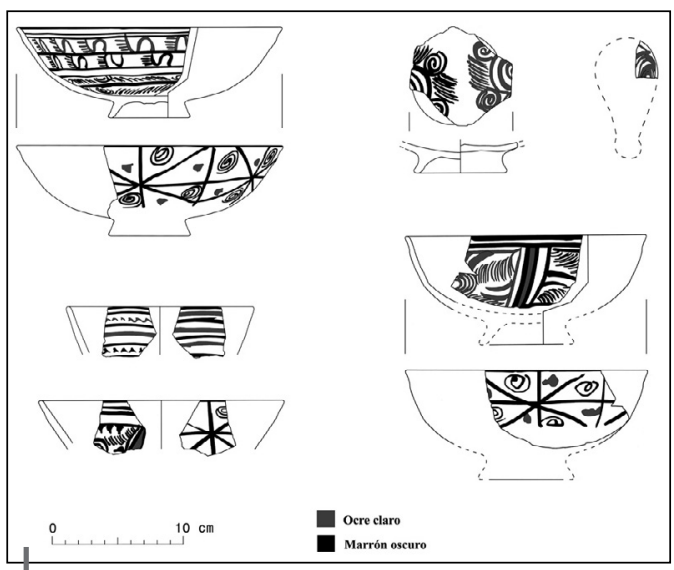

Fig. 8 - Alfar Cajamarca Cursivo Floral

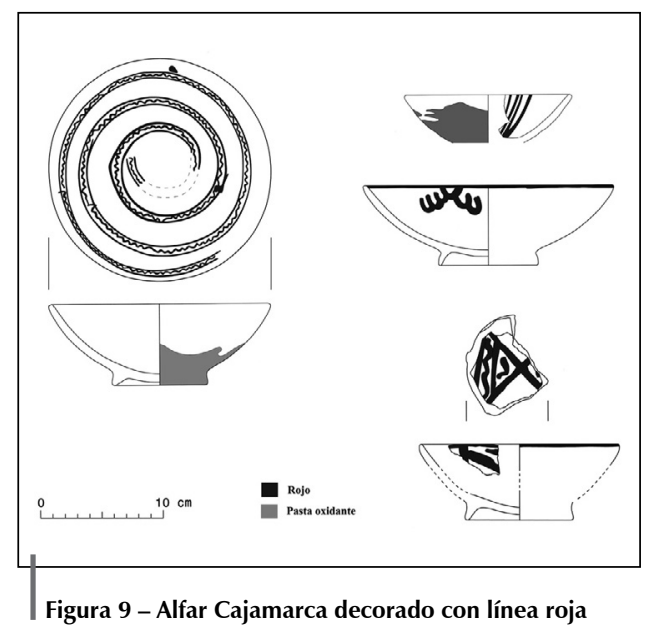


al interior, y otros un pequeño apéndice lateral modelado con forma de cabeza humana. Es importante precisar que estos diseños decorativos son totalmente ajenos a tradiciones cerámicas costeñas, y remiten más bien a formaciones serranas. La fuente exacta de estas tendencias decorativas nos es todavía, sin embargo, desconocida.

Finalmente, el alfar «Negro pulido» no solo es el más popular (59,5\%), sino también el más variado en cuanto a tipos de vasijas. Engloba a gran variedad de formas que incluyen: escudillas similares a las del alfar anterior, platos de base anular, cuencos, botellas de dos picos y asa puente, y botellas y cántaros con cara humana moldeada en el cuello (fig. 11). Algunas de estas formas (cuencos, por ejemplo) parecen derivadas de la tradición Moche Tardío. Otras (platos de base anular), parecen copias de formas Cajamarca. Las formas cerradas, por otro lado, remiten a estilos epigonales que alcanzaron amplia difusión en sitios de la costa y sierra peruana durante la segunda mitad del Horizonte Medio (Kroeber, 1925: lámina 46-j, 46-I; Flores Espinoza, 1959: foto 6; Menzel, 1977: figs. 51, 52 y 68; Topic \& Lange Topic, 1982-1985: figs. 6 y 7; Prümers, 2000: fig. 6).

En conclusión, la información ofrecida por la cerámica fina se inclina por la presencia de ocupantes de la sierra cercana, posiblemente del Valle de Cajamarca, en los edificios monumentales de Cerro Chepén. Esta información novedosa nos permite ahora esbozar un nuevo escenario de colapso para las comunidades Moche Tardío del valle, que está íntimamente relacionado con el problema de presión externa.

\section{DISCUSIÓN}

Las investigaciones desarrolladas por el PROCECHE en el centro fortificado de Cerro Chepén confirman la existencia de un enclave serrano en el corazón del valle bajo del Jequetepeque. Propongo que el sitio es producto de un movimiento expansivo emprendido alrededor del siglo VIII de nuestra era por un grupo altoandino, posiblemente asentado en los alrededores del valle de Cajamarca1. Los cajamarca siguieron el valle del Jequetepeque, que representa la ruta de

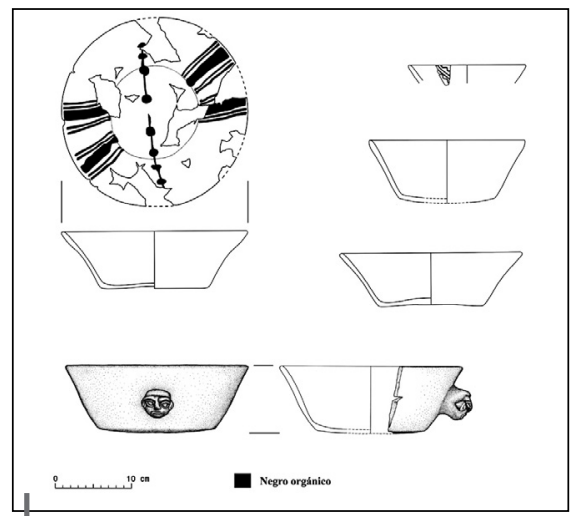

Figura 10 - Alfar Anaranjado pulido

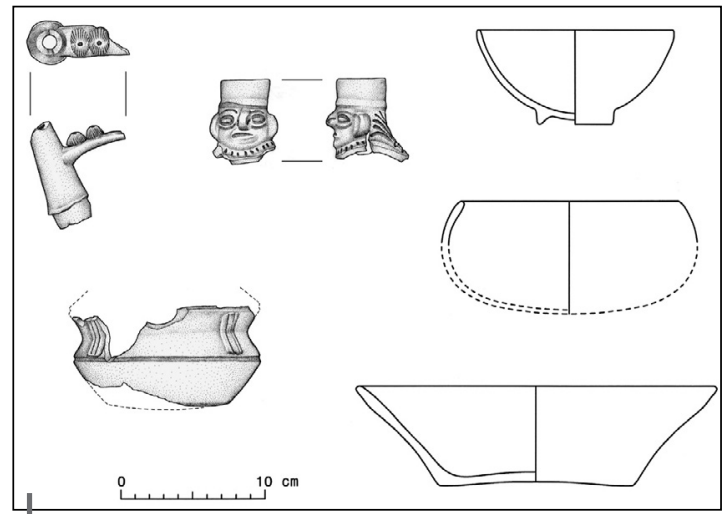

Figura 11 - Alfar Negro pulido

1 El más probable lugar de origen de esta expedición invasora se encuentra en el sitio de Guzmango Viejo, ubicado a $3100 \mathrm{msnm}$ en la provincia de Contumazá, a aproximadamente $48 \mathrm{~km}$ lineares al suroeste de la actual ciudad de Cajamarca. El sitio fue el centro de una de las warangas de la macroetnia Cajamarca durante el Horizonte Tardío (Julien, 1993: 251). El sitio, sin embargo, también fue ocupado durante el Horizonte Medio, y no solo ha 
comunicación más directa con la costa, como vía de expansión. Una vez que alcanzaron las tierras bajas, los cajamarca habrían consolidado su posición construyendo una plaza fuerte en un punto estratégico. El punto seleccionado fue la cima de un cerro que se levanta aproximadamente en el centro del valle bajo del Jequetepeque. Desde esta ubicación, los transgresores gozaban de acceso preferencial a agua y terrenos de cultivo, e inclusive ejercían un control visual sobre los cerros que rodean el valle, lugar donde se ubican los asentamientos de comunidades costeñas contemporáneas. Los cajamarca aparentemente también ejercieron control efectivo sobre algunos pobladores Moche, quienes tributaron vasijas domésticas, manufacturadas en claro estilo local, para su uso personal.

El esmerado arreglo defensivo del reducto cajamarquino, visible sobre todo en la muralla que rodea el Sector Monumental del sitio, sugiere que las relaciones con los nuevos vecinos costeños no fueron del todo amigables. Por el contrario, el ingenio y esfuerzo invertidos en dotar la muralla de mecanismos defensivos extremos (accesos restringidos, torreones, concentraciones de municiones) claramente indican que los habitantes del Sector Monumental de Cerro Chepén - entre ellos los ocupantes del Edificio IV - percibían que una seria amenaza se cernía sobre ellos. Tal como lo demostró el estudio de patrones de asentamiento desarrollado por Dillehay (2001) en el bajo Jequetepeque, el mismo sentimiento invadía a los pobladores Moche locales, quienes también se hallaban atrincherados en asentamientos fortificados.

En este momento es necesario precisar qué tan coherente es la propuesta de la invasión Cajamarca. A la luz de los conocimientos arqueológicos actuales, esta propuesta resulta absolutamente viable. Se sabe que la etnia Cajamarca forjó un poderoso cacicazgo, empezando en el siglo II de nuestra era, centrado en el valle que hoy ocupa la ciudad del mismo nombre (Julien, 1988). En la fase Cajamarca Medio (450 - 900 d. C.) esta cultura se expande fuera de los confines de su área nuclear, proyectando sus influencias hacia la zona del Marañón, la sierra Sur y, especialmente, los valle costeños (Terada \& Matsumoto, 1985: 87). En el valle bajo del río Chicama, vecino al sur del Jequetepeque, Leonard \& Russell (1993: 155) reconocen la presencia de asentamientos periféricos de la cultura Cajamarca que, sin embargo, no están fortificados ni trasgreden los límites de territorios Moche. Una situación muy similar es reportada por Shimada (1982: 179) en los valles de Zaña, Lambayeque y La leche durante el periodo Sicán Medio (850-1 100 d. C.). Para Shimada, la trasgresión serrana de las comarcas costeñas no habría sido posible debido a que las poblaciones locales se hallaban organizadas bajo un poderoso estado centralizado (1982: 185). Lo mismo se puede sugerir para el caso del valle de Chicama, revisado por Leonard \& Russel (1993). Este escenario, sin embargo, no es aplicable al caso del bajo Jequetepeque pues, como lo han demostrado Dillehay (2001) y Swenson (2004), en el Periodo Moche Tardío las poblaciones de la zona se hallaban disgregadas en comunidades autónomas.

Es importante precisar también cuál habría sido el rol que habría jugado la cultura Huari en la expansión Cajamarca. Como se ha mencionado varias veces a lo largo de este artículo, los edificios centrales del Sector Monumental de Cerro Chepén albergan espacios (galerías rodeando patios cuadrangulares, algunas de dos pisos) que han sido frecuentemente relacionadas con la tradición cultural ayacuchana. ¿Es posible que los Huari, y no los Cajamarca, sean los responsables de irrumpir en el territorio costeño?

En realidad, hay dos explicaciones posibles para esta aparente anomalía arquitectónica. En primer lugar, el modelo de galerías de dos pisos rodeando patios cuadrangulares presenta un fechado pre-Huari en la sierra Norte. Así lo demuestran las investigaciones desarrolladas por John Topic y Theresa Lange Topic en Huamachuco (1982-1985: 19), y algunas vasijas escultóricas de la cultura Recuay, asignables al Periodo Intermedio Temprano (ca. 200 a. C. - 600 d. C.), que representan espacios arquitectónicos de dos pisos (fig. 12). Es probable, entonces, que los arquitectos cajamarquinos hayan compartido el conocimiento sobre la construcción de galerías

reportado ingentes cantidades de platos de estilo Cajamarca Cursivo Floral, sino también un extraño alfar negro representado por platos de base anular (Julien, 1988: 198). Cabe destacar, que la arquitectura de Guzmango Viejo incluye galerías de varios pisos (Julien, 1988: 198). 


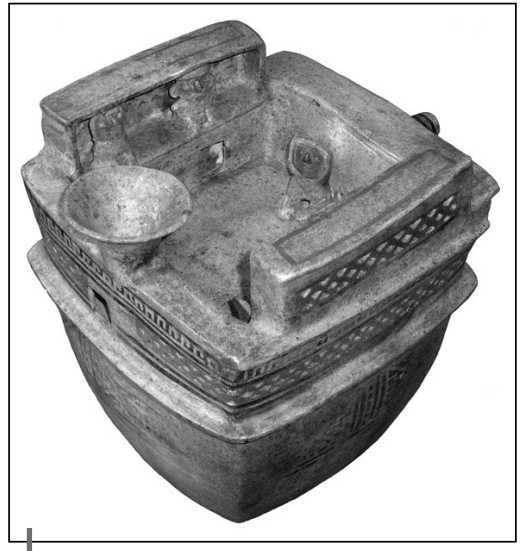

Figura 12 - Vasija escultórica Recuay con representación de edificios de dos pisos (Colección del Banco Central de Reserva del Perú)

de dos pisos con sus vecinos sureños, y lo hayan aplicado en sus construcciones. Este hecho, sin embargo, no está bien documentado en sitios del Intermedio Temprano del valle de Cajamarca.

La explicación alternativa considera una injerencia Huari, pero de carácter indirecto. Durante la expansión hacia la sierra Sur, ocurrida durante la fase Cajamarca Medio, los miembros de la etnia Cajamarca tomaron contacto con representantes de la cultura Huari que estaban, a su vez, emprendiendo su propio programa expansivo. Así lo atestiguan las ingentes cantidades de fragmentos cerámicos de estilo «Cursivo Floral» — semejantes a los hallados en el Edificio IV de Cerro Chepén- encontrados en sitios de avanzada Huari e inclusive la misma capital imperial (Menzel, 1964: 44; Ravines, 1968; Isbell, 1977; Julien, 1988: 226). La exacta relación que surgió del contacto entre las dos etnias serranas es aún materia de debate. Algunos autores (Lumbreras, 1974; Julien, 1988; Schreiber, 1992) sugieren que Cajamarca fue conquistada por los huari. Otros (Anders, 1986; Matsumoto, 1988), arguyen que la etnia Cajamarca fue lo suficientemente fuerte como para mantener su independencia. Opiniones conciliadoras sugieren que ambos grupos forjaron una alianza comercial, que en parte fue usada como catapulta para una expansión conjunta (Schaedel, 1985: 451; Matsumoto, 1988: 15; Shimada, 1994: 251). Los huari habrían erigido dos pequeños centros comercialesadministrativos con arquitectura de tipo grupo-patio en los alrededores del valle de Cajamarca (Williams \& Pineda, 1985). Según Julien (1988: 240) es en este momento en que los cajamarca asimilan el diseño de galerías rodeando patios cuadrangulares, plasmándolo en las estructuras centrales de sus asentamientos más representativos —incluyendo su capital regional, Cerro Coyor - durante la fase Cajamarca Medio.

La teoría del patrón arquitectónico aprendido del contacto con Huari ayuda a explicar algunas asociaciones materiales confusas detectadas en los edificios monumentales centrales de Cerro Chepén. Me refiero específicamente al caso de las vasijas cerradas propias al alfar «Negro pulido» que, como ya indiqué, repiten formas presentes en sitios de la costa y sierra peruana que denotan fuerte influencia Huari. También ayudaría a explicar la curiosa presencia de vasijas Huari policromo en el centro funerario de San José de Moro (Castillo, 2000). En este sitio, las vasijas Huari policromo aparecen asociadas a piezas asignables a nuestros alfares «Negro pulido», «Cajamarca decorado con línea roja gruesa», e inclusive platos «Cajamarca Cursivo Floral» dentro de tumbas de individuos prominentes. Yo sostengo que Cerro Chepén habría sido el sitio a través del cual se canalizaron todas estas piezas foráneas dentro del valle bajo del Jequetepeque2. Evidentemente, la recurrente asociación de cerámica Huari y Cajamarca dentro de contextos intrusivos al valle brinda apoyo a la teoría que sostiene que los Huari y Cajamarca, lejos de enfrentarse, se apoyaron mutuamente en sus empresas expansionistas.

\section{CONCLUSIÓN: COLAPSO CULTURAL EN EL BAJO JEQUETEPEQUE}

Habiendo sido definido el carácter de la ocupación del sitio de Cerro Chepén, y resuelta la incógnita acerca de las relaciones entabladas entre las tradiciones culturales Huari y Cajamarca,

2 Debo resaltar, sin embargo, que entre los 16500 tiestos recogidos en Cerro Chepén durante las campañas del 2003 y 2004 no figura uno solo que aluda al emblemático estilo polícromo Huari-estatal. Esta evidencia nos es útil, no obstante, para descartar arqueológicamente la presencia de administradores ayacuchanos en este enclave serrano. 
solo nos queda interpretar el rol que jugó el sitio en el colapso de las formaciones políticas Moche del bajo Jequetepeque. El escenario más factible de colapso lo hallamos en una revisión hecha por Bennett Bronson (1995) referente al rol que cumplieron los grupos nómades en el colapso de estados y civilizaciones antiguas. Según Bronson, un grupo beligerante expansivo no tiene necesariamente que arrasar militarmente a un estado para causar su colapso. A veces su sola presencia en la periferia del territorio estatal es motivo suficiente para causar el debilitamiento interno de las instituciones centrales de poder. Este debilitamiento surge cuando los gobernantes estatales se ven forzados a aumentar la carga impositiva sobre élites menores y población en general para enfrentar la amenaza e implementar acciones defensivas (construcción de muros, equipamiento de un ejército, alimentación de reclutas) (Bronson, 1995: 214). Estas acciones generalmente resultan impopulares y, como lo predice el modelo de colapso político de Tainter (1988), pueden incluso generar rebeldía social y negativas a pagar subsidios económicos.

Si bien los cajamarca no caen dentro de la categoría de «hordas barbáricas», ni los grupos locales habrían estado organizados bajo un «estado», considero que el modelo de Bronson es útil para explicar los eventos ocurridos en el Bajo Jequetepeque. Los transgresores Cajamarca podrían haber constituido fuerzas beligerantes lo suficientemente fuertes como para ejercer significativa presión sobre los grupos costeños. Su fuerza y capacidad organizativa queda demostrada en la majestuosidad de las obras defensivas del cerro, que fueron erigidas de una sola vez. Esta presión, ejercida desde el corazón mismo del territorio costeño, pudo aumentar significativamente los costos de manutención de las comunidades Moche locales. Como dato curioso, esta misma presión pudo haberles restado la posibilidad de recuperarse económicamente.

Estudios de patrones de asentamiento conducidos en Mesoamérica confirman que, cuando una región está ocupada por vecinos beligerantes, se forman amplias zonas desprovistas de ocupación separando los centros poblacionales en conflicto (Sanders et al., 1979: 103; Blanton et al., 1994: 99). Estas zonas vacías, comúnmente denominadas «tierra de nadie», muchas veces albergan tierras agrícolas de altísima calidad. Estas tierras simple y llanamente no pueden ser trabajadas por el riesgo que implica para los agricultores apartarse del resguardo de los asentamientos. En una situación de conflicto externo, entonces, grandes centros de población pueden ver su producción agrícola mermada. Según Dillehay (2001: 270), una situación de aprovechamiento ineficiente de terreno agrícola debido a conflicto entre comunidades habría imperado en el bajo Jequetepeque precisamente durante tiempos Moche Tardío. Esta situación se habría dado dentro de un marco de condiciones climáticas desfavorables (Shimada et al., 1991: 261; Shimada, 1994: 249).

Los habitantes de Cerro Chepén, que gozaban de un acceso preferencial a fuentes de agua y productos agrícolas, podrían haber mantenido su posición defensiva por más tiempo. La evidencia arqueológica indica, sin embargo, que esto no fue necesariamente así. En determinado momento de la secuencia Moche Tardío, el sitio y sus construcciones fueron abandonados. Una serie de fechados radiocarbónicos tomados de desechos orgánicos (maíz) asociados a los últimos eventos de ocupación en los edificios IV y VIII arrojaron fechas de $790 \pm 100$ (AA61865), $780 \pm$ 115 (AA61866) y $780 \pm 100$ (AA61870) años d. C.3. Estas fechas son comparables con los finales documentados de las ocupaciones de los sitios de Pampa Grande (Shimada, 1994: tabla 2), Galindo (Bawden, 1977: 410; Lockard, 2005: tabla 5. 4) e inclusive la zona urbana del sitio de huacas del Sol y de La Luna (Chapdelaine, 1998: 114). En el Valle de Jequetepeque, por otro lado, Edward Swenson (2004: tabla 6. 1) obtuvo un fechado prácticamente idéntico en el sitio Moche Tardío de Portachuelo de Charcape. La ausencia de señales de violencia acompañando el abandono de Cerro Chepén sugiere que éste fue planeado y pacífico. Aparentemente, los invasores Cajamarca fueron víctimas de las condiciones generadas por su propia irrupción en territorio ajeno. Las comunidades Moche Tardío habrían impuesto una férrea resistencia, forzando a los advenedizos a desistir de su proyecto expansionista.

3 Fechados AMS calibrados. Calculados con una desviación de 2 sigmas. El laboratorio estableció las correcciones correspondientes a variaciones en proporción de átomos de ${ }^{12} \mathrm{C}$ y ${ }^{13} \mathrm{C}$ propias a plantas de tipo $\mathrm{C}_{4}$. 
El escenario político del bajo Jequetepeque durante el periodo Moche Tardío ofrece una interesante imagen de lo que ocurre cuando dos sociedades complejas chocan por el control de un mismo territorio. Si uno de los poderes no es capaz de subyugar rápidamente a su oponente, se puede generar una «guerra de desgaste» con capacidad de debilitar significativamente a los bandos enfrentados. Si ninguno de los antagonistas cede en sus posiciones, el debilitamiento se puede prolongar hasta convertirse en causa de colapso de no solo uno, sino los dos grupos contrarios.

\section{Agradecimientos}

Quiero agradecer a la Dirección Académica de Investigación de la PUCP, especialmente a los profesores Carlos Chávez y Margarita Suárez, por el financiamiento parcial de las temporadas de campo de los años 2003 y 2004. Quisiera hacer mis agradecimientos extensivos a la Fundación Ethel-Jane Westfeldt Bunting y al Latin American and Iberian Institute de la Universidad de Nuevo México por haber proporcionado fondos para la campaña de mapeo de Cerro Chepén del año 2000. Muchos arqueólogos notables han ofrecido apoyo logístico o comentarios valiosos que han beneficiado directamente esta investigación. Entre ellos figuran Garth Bawden, James Boone, Jesús Briceño, Luis Jaime Castillo, Patricia Crown, Tom Dillehay, William Isbell, Carol Mackey, Krzysztof Makowski, Michael Moseley y Santiago Uceda. A ellos mi más sincero aprecio y reconocimiento. El proyecto se nutrió del talento y sacrificio de estudiantes de arqueología de la PUCP. Bárbara Carbajal, Marilyn Herrera, David Oshige y Elvis Mondragón fueron mis practicantes. Belén Gómez de la Torre, César Sara y Miguel Sordoméz también aportaron valioso esfuerzo e ideas durante el proceso de excavación. Muchas gracias a todos ellos. Gracias también a Luis Cáceres, a quien se debe en gran medida la confección del plano general del sitio. Los dibujos ejecutados con la técnica del puntillismo mostrados en este artículo son obra de Chiongwend Lhi.

Finalmente, quisiera agradecer a la Dra. Cecilia Bákula Budge por autorizar la publicación de la fotografía de la vasija Recuay ilustrada en la figura 12.

\section{Referencias citadas}

ALVA, W. \& DONNAN, C. B., 1993 - Tumbas Reales de Sipán, 229 p.; Los Angeles: Fowler Museum of Cultural History, University of California.

ANDERS, M. B., 1986 - Dual Organization and Calendars Inferred from the Planned Site of Azangaro - Wari Administrative Strategies. Tesis doctoral; Ithaca: Cornell University.

BAWDEN, G. L., 1977 - Galindo and the Nature of the Middle Horizon in Northern Coastal Peru. Tesis doctoral; Cambridge: Harvard University.

BAWDEN, G. L., 1982a - Galindo: a study in cultural transition during the Middle Horizon. In: Chan Chan: Andean Desert City (M. E. Moseley \& K. C. Day, eds.): 255-84; Albuquerque: University of New Mexico Press.

BAWDEN, G. L., 1982b - Community organization reflected by the household: a study of PreColumbian social dynamics. Journal of Field Archaeology, 9 (1): 165-81.

BAWDEN, G. L., 1994 - La paradoja estructural: la cultura Moche como ideología política. In: Moche: Propuestas y Perspectivas (S. Uceda \& E. Mujica, eds.): 389-412; Lima: Universidad Nacional de Trujillo - IFEA - FOMCIENCIAS. Actas del Primer Coloquio sobre la Cultura Moche (Trujillo, 12 al 16 de Abril de 1993).

BAWDEN, G. L., 1995 - The structural paradox: Moche culture as political ideology. Latin American Antiquity, 6 (2): 255-73.

BAWDEN, G. L., 1996 - The Moche, 375 p.; Cambridge: Blackwell Publishers Inc.

BAWDEN, G. L., 2001 - The symbols of Late Moche social transformation. In: Moche Art and Archaeology in Ancient Peru (J. Pillsbury, ed.): 285-305; Washington, D. C.: National Gallery of Art. 
BLANTON, R. E., KOWALEWSKI, S. A., FEINMAN, G. M. \& FINSTEN, L. M., 1994 - Ancient Mesoamerica: a Comparison of Change in three Regions, 284 p; Cambridge: Cambridge University Press.

BERDAN, F. F. \& SMITH, M. E., 1996 - Imperial strategies and core-periphery relations. In: Aztec Imperial Strategies (F. F. Berdan, R. E. Blanton, E. H. Boone, M. G. Hodge, M. E. Smith \& E. Umberger, eds.): 209-217; Washington, D.C.: Dumbarton Oaks Research Library and Collection.

BREWSTER-WRAY, C. C., 1983 - Spatial patterning and the function of a Huari architectural compound. In: Investigations of the Andean Past (D. H. Sandweiss, ed.): 122-135; Ithaca: Latin American Studies Program, Cornell University.

BRONSON, B., 1995 - The role of barbarians in the fall of states. In: The Collapse of Ancient States and Civilizations (N. Yoffee \& G. L. Cowgill, eds.): 196-218; Tucson: The University of Arizona Press.

BRUMFIEL, E. M. \& EARLE, T. K., 1990 - Specialization, exchange, and complex societies: an introduction. In: Specialization, Exchange, and Complex Societies (E. M. Brumfiel \& T. K. Earle, eds.): 1-9; Cambridge: Cambridge University Press.

CASTILLO, L. J., 1993 - Prácticas funerarias, poder e ideología en la sociedad Moche Tardía: El Proyecto Arqueológico San José de Moro. Gaceta Arqueológica Andina, 23: 67-82.

CASTILLO, L. J., 2000 - La presencia de Wari en San José de Moro. Boletín de Arqueología PUCP, 4: 143-179.

CASTILLO, L. J., 2003 - Los últimos mochicas en Jequetepeque. In: Moche: Hacia el Final del Milenio, Tomo II (S. Uceda \& E. Mujica, eds.): 65-123; Lima: Universidad Nacional de Trujillo y Pontificia Universidad Católica del Perú. Actas del Segundo Coloquio sobre la Cultura Moche (Trujillo, 1 al 7 de agosto de 1999).

CASTILLO, L. J. \& DONNAN, C. B., 1994 - La ocupación Moche de San José de Moro, Jequetepeque. In: Moche: Propuestas y Perspectivas (S. Uceda \& E. Mujica, eds.): 93146; Lima: Universidad Nacional de Trujillo - IFEA - FOMCIENCIAS. Actas del Primer Coloquio sobre la Cultura Moche (Trujillo, 12 al 16 de abril de 1993).

CASTILLO, L. J., NELSON, A. \& NELSON, C., 1997 - «Maquetas» mochicas de San José de Moro. Arkinka, 22: 120-128.

CHAPDELAINE, C., 1998 - Excavaciones en la zona urbana de Moche durante 1996. In: Investigaciones en la Huaca de la Luna (S. Uceda, E. Mujica \& R. Morales, eds.): 85-115; Trujillo: Facultad de Ciencias Sociales, Universidad Nacional de La Libertad.

COWGILL, G. L., 1995 - Onward and upward with collapse. In: The Collapse of Ancient States and Civilizations (N. Yoffee \& G. L. Cowgill, eds.): 244-247; Tucson: University of Arizona Press.

CULBERT, T. P., 1995 - The collapse of Classic Maya civilization. In: The Collapse of Ancient States and Civilizations (N. Yoffee \& G. L. Cowgill, eds.): 69-101; Tucson: University of Arizona Press.

DeMARRAIS, E., CASTILLO, L. J. \& EARLE, T. K., 1996 - Ideology, materialization, and power strategies. Current Anthropology, 37 (1): 15-31.

DILLEHAY, T. D., 2001 - Town and country in Late Moche times: a view from two northern valleys. In: Moche Art and Archaeology in Ancient Peru (J. Pillsbury, ed.): 259-283; Washington, D.C.: National Galery of Art. Studies in the History of Art 63, Center for Advanced Studies in the Visual Arts, Symposium Papers XL.

DONNAN, C. B., 1975 - An ancient Peruvian architectural model. The Masterkey, 49 (1): 20-29.

DONNAN, C. B., 1986 - The city walls at Pacatnamu. In: The Pacatnamú Papers, vol. I (C. B. Donnan \& G. A. Cock, eds.): 47-62; Los Angeles: Fowler Museum of Cultural History, University of California.

DONNAN, C. B., 2003 - Tumbas con entierros en miniatura: un nuevo tipo funerario Moche. In: Moche: Hacia el Final del Milenio, tomo II (S. Uceda \& E. Mujica, eds.): 43-78; Lima: Universidad Nacional de Trujillo y Pontificia Universidad Católica del Perú. Actas del Segundo Coloquio sobre la Cultura Moche (Trujillo, 1 al 7 de agosto de 1999). 
DONNAN, C. B. \& CASTILLO, L. J., 1992 - Finding the tomb of a Moche priestess. Archaeology, 45 (6): 38-42.

DONNAN, C. B. \& CASTILLO, L. J., 1994 - Excavaciones de tumbas de sacerdotisas Moche en San José de Moro. In: Moche: Propuestas y Perspectivas (S. Uceda \& E. Mujica, eds.): 415-424; Lima: Universidad Nacional de Trujillo, IFEA, FOMCIENCIAS. Actas del Primer Coloquio sobre la Cultura Moche (Trujillo, 12 al 16 de abril de 1993).

DONNAN, C. B. \& McCLELLAND, D., 1999 - Moche Fineline Painting: its Evolution and its Artists, 319 p.; Los Angeles: Fowler Museum of Cultural History, University of California.

ELING, H., 1987 - The Role of Irrigation Networks in Emerging Societal Complexity During Late Pre-Hispanic Times, Jequetepeque Valley, North Coast, Perú. Tesis doctoral; Austin: The University of Texas.

FLORES ESPINOZA, G. I., 1959 - El sitio arqueológico de Wari Willka, Huancayo. Época Prehispánica, 1: 177-186. Actas y Memorias del Segundo Congreso Nacional de Historia del Perú.

ISBELL, W. H., 1977 - The Rural Foundation for Urbanism: Economic and Stylistic Interaction between Rural and Urban Communities in Eight Century Perú, 186 p.; Urbana: University of Illinois Press.

ISBELL, W. H., 1989 - Honcopampa: was it a Huari administrative centre? In: The Nature of Wari. A Reappraisal of the Middle Horizon Period in Perú (R. M. Czwarno, F. M. Meddens \& A. Morgan, eds.): 98-114; Oxford: BAR International Series 525.

ISBELL, W. H., 1991 - Huari administration and the Orthogonal Cellular Architecture Horizon. In: Huari Administrative Structure. Prehistoric Monumental Architecture and State Government (W. H. Isbell \& G. F. McEwan, eds.): 293-315; Washington, D. C.: Dumbarton Oaks Research Library and Collection.

JULIEN, D. G., 1988 - Ancient Cuismancu: Settlement and Cultural Dynamics in the Cajamarca Region of the North Highlands of Perú, 200 B. C.-A. D. 1532. Tesis doctoral; Austin: The University of Texas.

JULIEN, D. G., 1993 - Late Pre-Inkaik ethnic groups in highland Peru: An archaeologicalethnohistorical model of the political geography of the Cajamarca region. Latin American Antiquity, 4 (3): 246-273.

KAUFMAN, H., 1995 - The collapse of ancient states and civilizations as an organizational problem. In: The Collapse of Ancient States and Civilizations (N. Yoffee \& G. L. Cowgill, eds.): 219-35; Tucson: The University of Arizona Press.

KROEBER, A. L., 1925 - The Uhle Pottery Collections from Moche. University of California Publications in American Archaeology and Ethnology, 21 (5): 191-234.

LARCO HOYLE, R., 2001 [1939] - Los Mochicas, t. I, 333 p.; Lima: Museo Arqueológico Rafael Larco Herrera, Servicios Editoriales del Perú SAC.

LEONARD, B. L. \& RUSSELL, G. S., 1993 - Cerámica Cajamarca en la parte baja del valle de Chicama. In: Actas del IX Congreso Peruano del Hombre y la Cultura Andina (S. Arréstegui, ed.): 151-165; Cajamarca: Universidad Nacional de Cajamarca.

LOCKARD, G. D., 2005 - Political Power and Economy at the Archaeological Site of Galindo, Moche Valley, Peru. Tesis doctoral; Albuquerque: The University of New Mexico.

LUMBRERAS, L. G., 1974 - The Peoples and Cultures of Ancient Perú, 248 p.; Washington, D. C.: Smithsonian Institution Press.

MATSUMOTO, R., 1988 - The Cajamarca Culture: its Evolution and Interaction with Coastal Polities, 39 p. Manuscrito.

MATSUMOTO, R., 1994 - Dos modos de proceso sociocultural: el Horizonte Temprano y el Periodo Intermedio Temprano en el valle de Cajamarca. In: El Mundo Ceremonial Andino (L. Millones \& Y. Onuki, eds.): 167-197; Lima: Editorial Horizonte.

MENZEL, D., 1964 - Style and Time in the Middle Horizon. Nawpa Pacha, 2: 1-105. 
MENZEL, D., 1977 - The Archaeology of Ancient Perú and the Work of Max Uhle, 135 p.; Berkeley: R. H. Lowie Museum of Anthropology, University of California.

NARVÁEZ, A., 1994 - La Mina: una tumba Moche I en el valle de Jequetepeque. In: Moche: Propuestas y Perspectivas (S. Uceda \& E. Mujica, eds.): 59-81; Lima: Universidad Nacional de Trujillo, IFEA, FOMCIENCIAS. Actas del Primer Coloquio sobre la Cultura Moche (Trujillo, 12 al 16 de abril de 1993).

PRÜMERS, H., 2000 - «El Castillo» de Huarmey: una plataforma funeraria del Horizonte Medio. Boletín de Arqueología PUCP, 4: 289-312.

RAVINES, R., 1968 - Un depósito de ofrendas del Horizonte Medio en la sierra central del Perú. Ñawpa Pacha, 6: 19-45.

REICHLEN, H. \& REICHLEN, P., 1970 - Reconocimientos arqueológicos en los Andes de Cajamarca. In: 100 Años de Arqueología en el Perú (R. Ravines, ed.): 463-501; Lima: Instituto de Estudios Peruanos, Edición de Petróleos del Perú. Fuentes e Investigaciones para la Historia del Perú, 3.

RUCABADO, J. \& CASTILLO, L. J., 2003 - El Periodo Transicional en San José de Moro. In: Moche: Hacia el Final del Milenio, tomo II (S. Uceda \& E. Mujica, eds): 15-42; Lima: Universidad Nacional de Trujillo, Pontificia Universidad Católica del Perú. Actas del Segundo Coloquio sobre la Cultura Moche (Trujillo, 1 al 7 de agosto de 1999).

SANDERS, W. T., PARSONS, J. R. \& SANTLEY, R. S., 1979 - The Basin of Mexico: Ecological Processes in the Evolution of a Civilization, 561 p.; New York: Academic Press.

SANTLEY, R.S., KILLION, T.W. \& LYCETT, M.T., 1986 - On the Maya collapse. Journal of Anthropological Research, 42: 123-159.

SCHAEDEL, R. P., 1985 - The transition from chiefdom to state in northern Peru. In: Development and Decline: The Evolution of Sociopolitical Organization (H. J. M. Claessen, P. van de Velde \& M. E. Smith, eds.): 156-69; South Hadley: Bergin and Garvey.

SCHREIBER, K. J., 1992 - Wari Imperialism in Middle Horizon Peru, 332 p.; Ann Arbor: Museum of Anthropology, University of Michigan. Anthropological Papers, 87.

SHIMADA, I., 1982 - Horizontal archipelago and coast-highland interaction in north Peru: archaeological models. In: El Hombre y su Ambiente en los Andes Centrales (L. Millones \& H. Tomoeda, eds.): 137-210; Osaka: National Museum of Ethnology. Senri Ethnological Studies, 10.

SHIMADA, I., 1994 - Pampa Grande and the Mochica Culture, 323 p.; Austin: University of Texas Press.

SHIMADA, I., SCHAAF, C. B., THOMPSON, L. G. \& MOSLEY-THOMPSON, E., 1991 - Cultural impacts of severe droughts in the prehistoric Andes: application of a 1 500-year ice core precipitation record. World Archaeology, 22 (3): 247-270.

STRONG, W. D., 1947 - Finding the tomb of a warrior-god. National Geographic Magazine, 91: 453-482.

SWENSON, E. R., 2004 - Ritual and Power in the Urban Hinterland: Religious Pluralism and Political Decentralization in Late Moche Jequetepeque, Peru. Tesis doctoral; Chicago: The University of Chicago.

TAINTER, J. A., 1988 - The Collapse of Complex Societies, 250 p.; Cambridge: Cambridge University Press.

TELLO, R., ARMAS, J. \& CHAPDELAINE, C., 2003 - Prácticas funerarias Moche en el complejo arqueológico Huacas del Sol y de la Luna. In: Moche: Hacia el Final del Milenio, tomo II (S. Uceda \& E. Mujica, eds.): 151-187; Lima: Universidad Nacional de Trujillo, Pontificia Universidad Católica del Perú. Actas del Segundo Coloquio sobre la Cultura Moche (Trujillo, 1 al 7 de agosto de 1999).

TERADA, K. \& MATSUMOTO, R., 1985 - Sobre la cronología de la tradición Cajamarca. In: Historia de Cajamarca I. Arqueología (F. Silva Santisteban, W. Espinoza Soriano \& R. Ravines, eds.): 67-89; Cajamarca: Instituto Nacional de Cultura Cajamarca, Corporación de Desarrollo de Cajamarca. 
THOMPSON, L. G., 1980 - Glaciological investigations of the tropical Quelccaya ice cap, Peru. Journal of Glaciology, 25 (91): 69-84.

THOMPSON, L. G. \& MOSLEY-THOMPSON, E., 1987 - Evidence of abrupt climatic change during the last 1500 years recorded in ice cores from the tropical Quelccaya ice cap, Peru. In: Abrupt Climatic Change (W. H. Berger \& L. D. Labeyrie, eds.): 99-110; Norwell: D. Reidel Publishing Company. NATO ASI Series C, vol. 216.

THOMPSON, L. G., HASTENRATH, S. \& MORALES ARNAO, B., 1979 - Climatic ice core records from the Tropical Quelccaya ice cap. Science, 203: 1240-1243.

THOMPSON, L. G., MOSLEY-THOMPSON, E., BOLZAN, J. F. \& KOCI, B. R., 1985 - A 1 500year record of tropical precipitation in ice cores from the Quelccaya ice cap, Peru. Science, 229: 971-973.

THOMPSON, L. G., MOSLEY-THOMPSON, E., DANSGAARD, W. \& GROOTES, P. M., 1986 The Little Ice Age as recorded in the stratigraphy of the tropical Quelccaya ice cap. Science, 234: 361-364.

THOMPSON, L. G., MOSLEY-THOMPSON, E., GROOTES, P. M., POURCHET, M. \& HASTENRATH, S., 1984 - Tropical glaciers: potential for ice core paleoclimatic reconstructions. Journal of Geophysical Research, 89 (D3): 4638-4646.

TOPIC, J. R. \& LANGE TOPIC T., 1982-1985 - El Horizonte Medio en Huamachuco. Revista del Museo Nacional, 47: 13-52

TRIGGER, B. G., 1990 - Monumental architecture: a thermodynamic explanation of symbolic behavior. World Archaeology, 22 (2): 119-131.

UCEDA, S., 1997 - Esculturas en miniatura y una maqueta en madera. In: Investigaciones en la Huaca de la Luna 1995 (S. Uceda, E. Mujica \& R. Morales, eds.): 151-176; Trujillo: Facultad de Ciencias Sociales, Universidad Nacional de La Libertad.

WILLIAMS, C. \& PINEDA, J., 1985 - Desde Ayacucho hasta Cajamarca: formas arquitectónicas con filiación Wari. Boletín de Lima, 40: 55-61.

YOFFEE, N., 1979 - The decline and rise of Mesopotamian civilization: an ethnoarchaeological perspective on the evolution of social complexity. American Antiquity, 44 (1): 5-35. 